\title{
A Mathematical Images Group Model to Estimate the Sound Level in a Close-Fitting Enclosure
}

\author{
Michael J. Panza \\ Mechanical Engineering, Gannon University, 109 University Square, Erie, PA 16541, USA \\ Correspondence should be addressed to Michael J. Panza; panza@gannon.edu
}

Received 11 January 2014; Revised 9 May 2014; Accepted 21 May 2014; Published 12 June 2014

Academic Editor: Andrew Peplow

Copyright ( 2014 Michael J. Panza. This is an open access article distributed under the Creative Commons Attribution License, which permits unrestricted use, distribution, and reproduction in any medium, provided the original work is properly cited.

\begin{abstract}
This paper describes a special mathematical images model to determine the sound level inside a close-fitting sound enclosure. Such an enclosure is defined as the internal air volume defined by a machine vibration noise source at one wall and a parallel reflecting wall located very close to it and acts as the outside radiating wall of the enclosure. Four smaller surfaces define a parallelepiped for the volume. The main reverberation group is between the two large parallel planes. Viewed as a discrete line-type source, the main group is extended as additional discrete line-type source image groups due to reflections from the four smaller surfaces. The images group approach provides a convergent solution for the case where hard reflective surfaces are modeled with absorption coefficients equal to zero. Numerical examples are used to calculate the sound pressure level incident on the outside wall and the effect of adding high absorption to the front wall. This is compared to the result from the general large room diffuse reverberant field enclosure formula for several hard wall absorption coefficients and distances between machine and front wall. The images group method is shown to have low sensitivity to hard wall absorption coefficient value and presents a method where zero sound absorption for hard surfaces can be used rather than an initial hard surface sound absorption estimate or measurement to predict the internal sound levels the effect of adding absorption.
\end{abstract}

\section{Introduction}

Many studies have been done on the insertion loss of enclosures including the effect of reverberant buildup inside enclosures as well as the sound transmission loss of the enclosure surfaces. As the focus of this paper is the internal sound field only, analytical studies relating, in some significant way, to this are considered. Tweed and Tree [1] focus on the insertion loss of the enclosure (i.e., difference in sound pressure level at the same position outside enclosure) and compare the prediction from Jackson [2], Junger [3], and Ver [4]. Each of these three studies considers a plane wave radiated by the noise source. Jackson [2] considers infinite wall panel sound transmission loss and Junger [3] considers a finite panel sound transmission loss. Ver [4] considers three frequency regions relative to panel fundamental natural frequency and the acoustic frequency of lumped air space between source and wall panel. Tweed and Tree [1] conclude that all three methods give differences large enough to cause concerns in accurate enclosure design. Osman [5] uses the diffuse field internal sound pressure level model presented in Embleton [6] for large room reverberation and applies it to close-fitting parallelepipeds for source and enclosure separated by only one meter. The method is applied to several sizes of diesel engine generator sets. Sgard et al. [7] consider large and not close-fitting enclosures and divide the internal volume into several cavities. They use an image series with nonzero sound absorption to improve convergence for the cavities pressure. These cavity pressures are then used as inputs in a statistical energy analysis model from cavities to enclosure panels to exterior radiation. All of the above papers look at the entire enclosure insertion loss from the standpoint of effectiveness of prediction method. Thus they focus on the combined effect of internal reverberation and surface transmission loss and not specifically on how the internal sound pressure level is modeled. 
The main focus of this paper is the effect of the internal reverberation model on the interior sound pressure level. For the model in this paper, the main reverberation is considered to be between the noise radiating surface and its parallel wall (referred to as the front wall) which in turn would radiate through the wall to the exterior of the enclosure. This is referred to as the main images group and can be viewed as discrete line-type source. The internal sound field is modeled with an infinitesimal amount of machine surface radiating direct sound incident to the front wall and multiplereflected reverberant sound composed of the image sum produced by machine surface and cab wall, one-half of which is incident to the front wall. The infinitesimal pressure intensity is integrated over the machine side surface. This method of noise radiation from rectangular machine sources is patterned after Hohenwarter [8]. The small enclosure side surfaces are referred to as bulkheads, the small bottom surface as floor, and the small top surface as ceiling. The main reverberant group part described above is amplified by considering its discrete line-type images from the two bulkheads' reflections, its discrete line-type images from the floor/ceiling reflections, and its discrete line-type images from combined bulkheads, floor/ceiling reflections.

The images group approach used here allows an untreated enclosure to contain no sound absorption and be represented by perfectly reflecting surfaces with zero sound absorption coefficient. It also allows supplier measured sound absorption coefficient value to be used for a treated enclosure case. Thus, as is not the case with the standard large room diffuse field reverberation model presented in Embleton [6], the untreated internal surface condition does not have to be measured or estimated for an actual enclosure. This is important because the theoretical sound absorption coefficient $\alpha$ of acoustically hard steel or fiberglass surfaces that comprise the cab interior can typically range from 0.001 to 0.05 . Additionally, the diffuse field model gives a reverberant field contribution inversely proportional to $\alpha$.

The reverberant sound intensity incident to and perpendicular to the front wall is one-half of the internal reverberant pressure intensity due to space averaging over all angles of incident from the multiple images. This is the standard model for reverberation in enclosures as given by Embleton [6]. The images group method gives a sound pressure level field that is essentially the same in the space between machine and enclosure. It puts emphasis on the distance between machine and enclosure.

The model presented here is intended for enclosures that are large enough relative to the primary frequency content of the machine surface. The effect of any one isolated dominant standing wave is considered to be small and is not included in the model. The octave band A-weighted frequency spectrum of the machine radiating surface is expected to be relatively broadband with primary A-weighted sound power contributions from 500 to $8000 \mathrm{~Hz}$. Such a situation is commonplace covering a large range of applications. The model can be used in the sense of overall $\mathrm{dBA}$ by using the overall sound power level $\mathrm{dBA}$ value of the machine radiating surface and an Aweighted dBA value for surface sound absorption coefficients by using the source sound power as the weighting factor.

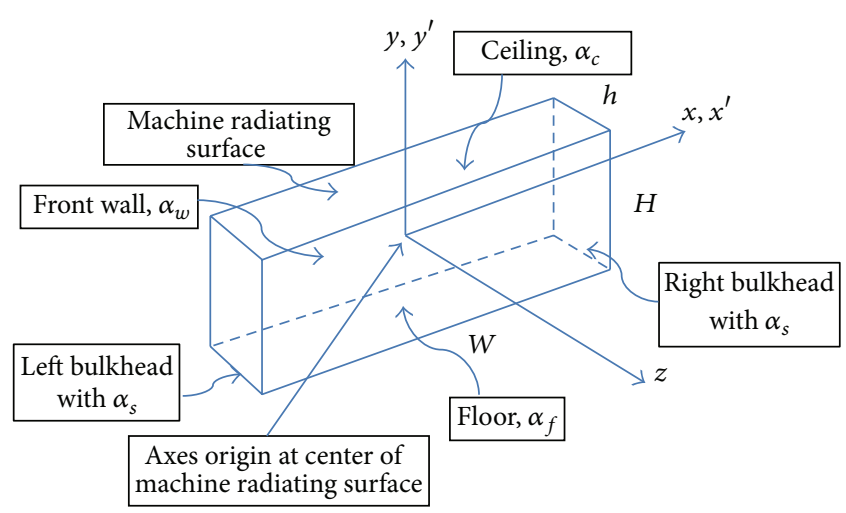

FIGURE 1: Internal acoustic volume: reverberation occurs in the prismatic region between the machine surface radiating surface and enclosure front wall.

The model can also be used for one-third or octave bands by using the one-third or octave band source A-weighted sound power level values and the particular surface sound absorption coefficient values in each one-third or octave band. For sources with significant A-weighted contributions somewhat below $500 \mathrm{~Hz}$, especially when specific pure tones may be present, the one-third or octave estimates should be viewed in a frequency average sense in these bands. The numerical examples used in this paper satisfy these criteria. If one desires, the images group model estimates in these lower frequency bands could then be enhanced with a specific analytical or numerical finite difference method to reveal more peaks across these lower bands for sources more inclined to contain significant lower frequency pure tones.

\section{Internal Sound Field Model}

Figures 1-4 give a layout of the coordinate system and the image groups.

Figure 1 is a 3 -dimensional view of the internal enclosure volume between the machine side surface and the front wall. The main reverberation is along the $z$-axis between the machine side surface and the front wall parallel surfaces.

Figure 2 is a plan view of the $x-z$ plane showing the multiple machine surface, enclosure front wall images, and the infinitesimal amount of sound power $d I I_{e}$ radiated from the machine surface at position $\left(x^{\prime}, y^{\prime}\right)$ to wall surface at position $(x, y)$, which has a random incidence sound absorption coefficient $\alpha_{w}$. The stars refer to the location of the point source image after a particular number of machine radiating surface, enclosure front wall multiple reflections. Seven-point stars indicate that the final reflection is from the ceiling and five-point stars indicate that the final reflection is from the enclosure front wall. The infinite line of point source images is referred to as a discrete line-type source.

Figure 3 is an elevation view of the $x-y$ plane showing the image groups for the two-side bulkheads, the floor, and the ceiling. The main group 1 from Figure 2 is in the center and it produces image groups $\mathrm{A}, \mathrm{B}, \mathrm{C}$, and D as described in the figure. Each image as a part of a group is a discrete line-type 


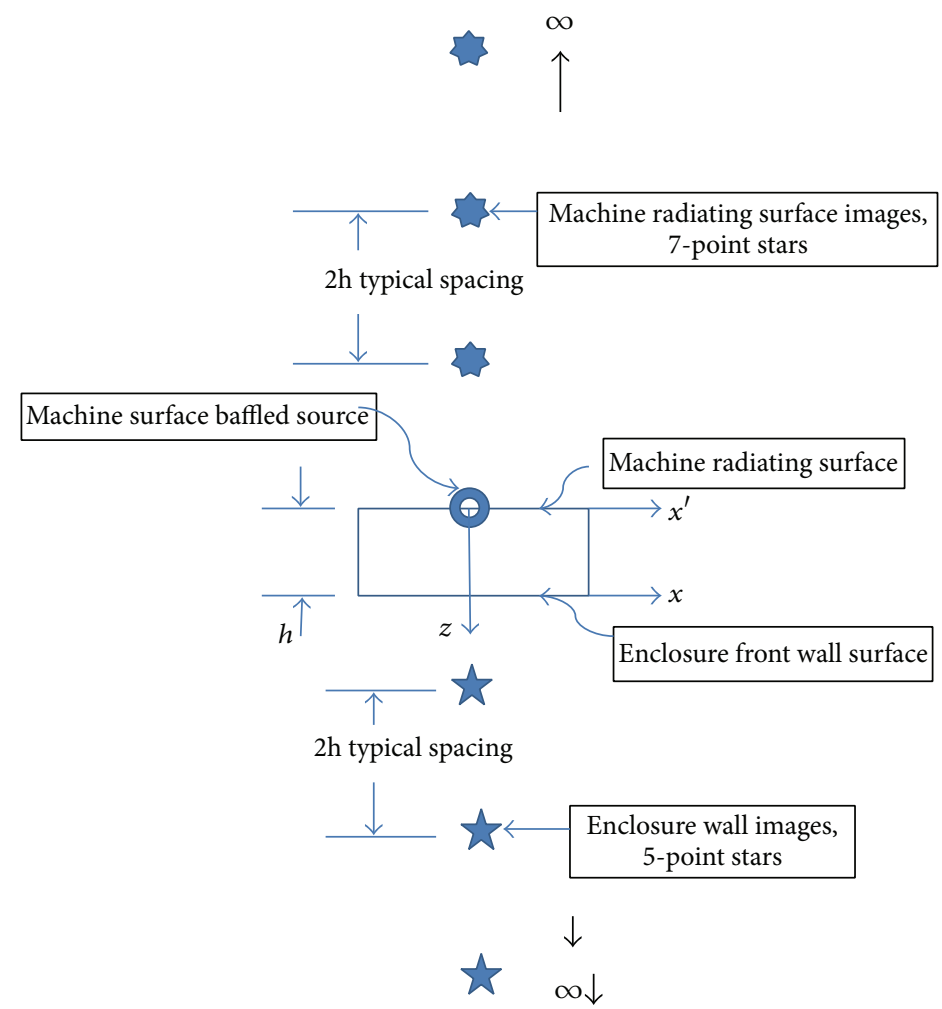

FIGURE 2: Plan view of the $x-z$ plane showing the multiple machine radiating surface and front wall images of the infinitesimal point sources at the machine radiating surface. This is group number 1, the main reverberation. Since machine radiating surface is a baffled source, each image is a double spherical source. The overall effect can be viewed as a discrete line-type source.

source. Each group consists of an infinite number of line-type images. Figure 4 gives a flow chart showing the development of the image groups from the main group.

The infinitesimal power $d I I_{e}$ is radiated from an infinitesimal area $d x^{\prime} d y^{\prime}$, which is baffled by the adjacent hard surface of the machine. Consider $d I I_{e}=I I_{e} / W H$, where $I I_{e}$ is the total sound power emanating from the machine radiating surface. The infinitesimal amount of reverberant mean square sound pressure at the front wall is given by the sum of all of the image sources at the engine surface and cab wall. The factor of 2 accounts for the machine surface baffling effect. Consider

$$
\begin{aligned}
d p_{R}^{2}= & 2 \sum_{n=1}^{\infty} \frac{\rho c d I I_{e}\left(1-\alpha_{w}\right)^{n}}{4 \pi\left[r^{2}+(2 n h-h)^{2}\right]} \\
& \text { Machine Surface } \\
+ & 2 \sum_{n=1}^{\infty} \frac{\rho c d I I_{e}\left(1-\alpha_{w}\right)^{n}}{4 \pi\left[r^{2}+(2 n h+h)^{2}\right]}
\end{aligned}
$$

\section{Front Wall}

where $r^{2}=\left(x-x^{\prime}\right)^{2}+\left(y-y^{\prime}\right)^{2}$ and $h$ is the distance between the machine radiating surface and the enclosure front wall.
The infinitesimal amount of mean square sound pressure at the front wall due to the direct sound from the machine surface is given by

$$
d p_{D}^{2}=\frac{\rho c d I I_{e}}{4 \pi\left(r^{2}+H^{2}\right)} .
$$

The origin of the coordinate system is at the center of the machine radiating surface and $b=H / 2, a=W / 2$. The total mean square pressure at the front wall and incident to the front wall due to the entire machine surface is the integral over the surface

$$
p_{w}^{2}(x, y)_{\text {incident }}=\int_{-b}^{b} \int_{-a}^{a}\left(d p_{D}^{2}+\frac{d p_{R}^{2}}{2}\right) d x^{\prime} d y^{\prime},
$$

where the $1 / 2$ is for only half of the reverberant pressure squared incident to the front wall where it can be transmitted through the wall.

Assuming that the distance $h$ between the machine surface and the front wall is much smaller than the size of the engine surface dimensions, the double integral for direct sound from machine surface can be given by the relationship for a plane source of uncorrelated noise sources. Consider

$$
p_{w \text { incident direct }}^{2}=\frac{\rho c I I_{e}}{4 a b},
$$




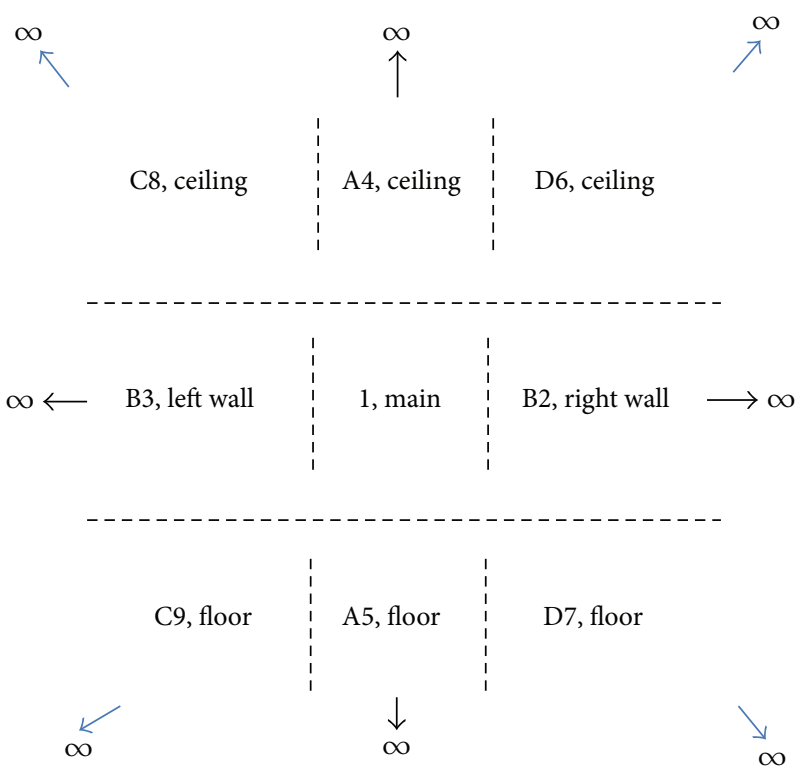

FIGURE 3: Plan view of the $x-y$ plane showing image groups. (Main group 1 from Figure 2 for multiple machine radiating surface and enclosure front wall images. It can be viewed as a discrete line-type source.) (Group A) The ceiling (A4) and floor (A5) images of the main group 1. (Group B) The left bulkhead (B3) and right bulkhead (B2) images of the main group 1. (Group C) The ceiling (C8) images of the left bulkhead image group (B3) with ceiling image group (A4). The floor (C9) images of the left bulkhead image group (B3) with floor image group (A5). (Group D) The ceiling (D6) images of the right bulkhead image group (B2) with ceiling image group (A4). The floor (D7) images of the right bulkhead image group (B2) with floor image group (A5). Each group can be viewed as distinct line-type sources perpendicular to the layout page. The main group is one distinct line-type source.

which is independent of $x, y$ position on the wall. $I I_{e}$ is the power radiated by the machine surface.

The reverberant component incident to the front wall is given by

$$
\begin{aligned}
& p_{w}^{2}(x, y)_{\text {incident reverb }} \\
& =\frac{2 \rho c\left(I I_{e} / 4 a b\right)}{2(4 \pi)} \sum_{n=1}^{\infty}\left(1-\alpha_{w}\right)^{n} \\
& \quad *\left[\int_{-b}^{b} \int_{-a}^{a} \frac{d x^{\prime} d y^{\prime}}{\left(x-x^{\prime}\right)^{2}+\left(y-y^{\prime}\right)^{2}+(2 n h-h)^{2}}\right. \\
& \left.\quad+\int_{-b}^{b} \int_{-a}^{a} \frac{d x^{\prime} d y^{\prime}}{\left(x-x^{\prime}\right)^{2}+\left(y-y^{\prime}\right)^{2}+(2 n h+h)^{2}}\right] .
\end{aligned}
$$

The integrals for $d x^{\prime}$ are of the form

$$
\int_{-a}^{a} \frac{d x^{\prime}}{\left(x-x^{\prime}\right)^{2}+\beta_{i}^{2}}
$$

which has a closed form solution.

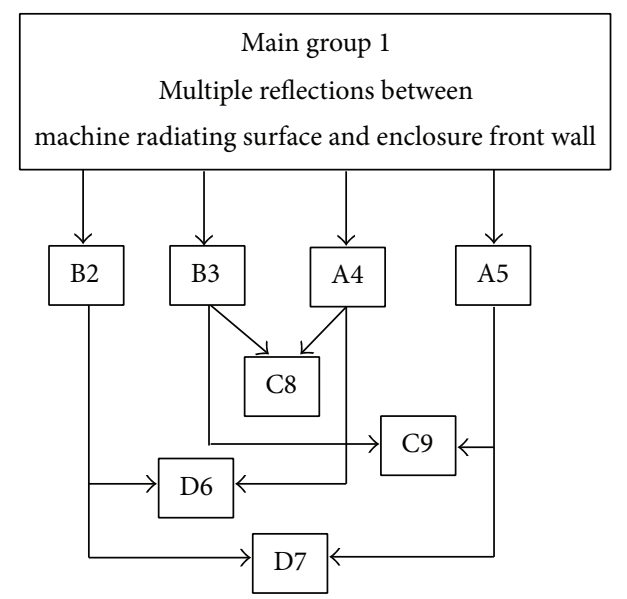

FIGURE 4: Image group flow chart.

Letting $x-x^{\prime}=u \rightarrow d x^{\prime}=-d u$, (6) becomes

$$
\begin{aligned}
-\int_{x+a}^{x-a} & \frac{d u}{u^{2}+\beta_{i}^{2}}=\left.\frac{1}{\beta_{i}} \tan ^{-1}\left(\frac{u}{\beta_{i}}\right)\right|_{x-a} ^{x+a} \\
= & \frac{1}{\beta_{i}}\left[\tan ^{-1}\left(\frac{x+a}{\beta_{i}}\right)-\tan ^{-1}\left(\frac{x-a}{\beta_{i}}\right)\right],
\end{aligned}
$$

where $\beta_{2}=\sqrt{\left(y-y^{\prime}\right)^{2}+(2 n h-h)^{2}}, \quad \beta_{3}=$ $\sqrt{\left(y-y^{\prime}\right)^{2}+(2 n h+h)^{2}}$.

Equation (5) becomes

$$
\begin{aligned}
& p_{w}^{2}(x, y)_{\text {incident reverb }} \\
& =\frac{\rho c I I_{e}}{16 \pi a b} \sum_{n=1}^{\infty}\left(1-\alpha_{w}\right)^{n} \\
& \quad *\left\{\int_{-\mathrm{b}}^{b} \frac{1}{\beta_{2}}\left[\tan ^{-1}\left(\frac{x+a}{\beta_{2}}\right)-\tan ^{-1}\left(\frac{x-a}{\beta_{2}}\right)\right] d y^{\prime}\right. \\
& \left.\quad+\int_{-\mathrm{b}}^{b} \frac{1}{\beta_{3}}\left[\tan ^{-1}\left(\frac{x+a}{\beta_{3}}\right)-\tan ^{-1}\left(\frac{x-a}{\beta_{3}}\right)\right] d y^{\prime}\right\} .
\end{aligned}
$$

The integral for $d y^{\prime}$ is solved numerically for an upper sum limit of $N$ images.

Equation (8) is for the main reverberation between machine surface and front wall. It is determined for $x=0$, $y=0$ location and identified as group number 1 in Figures 3 and 4. Additional parts from floor and ceiling reflections, left bulkhead and right bulkhead reflections, and combined floor, ceiling, and bulkheads are also determined from (8). These contributions are modeled as images of the main group number 1 machine surface and front wall result.

(i) Floor reflection of main part group 1 to produce group A5 in Figures 3 and $4 ; x=0, y=H$

$$
p_{w}^{2}(0, H)_{\text {incident reverb }} \text {. }
$$


(ii) Ceiling reflection of main part group 1 to produce group A4 in Figures 3 and 4; $x=0, y=H$

$$
p_{w}^{2}(0, H)_{\text {incident reverb }}\left(1-\alpha_{c}\right) \text {. }
$$

(iii) Right wall and left wall reflection of main part group 1 to produce groups B2 and B3 in Figures 3 and 4; $x=$ $W, y=0$

$$
p_{w}^{2}(W, 0)_{\text {incident reverb }}\left(1-\alpha_{s}\right) .
$$

(iv) Floor reflection of right wall and left wall groups B2, B3 combined with group A5 to produce groups D7 and C9 in Figures 3 and $4 ; x=W, y=H$

$$
p_{w}^{2}(W, H)_{\text {incident reverb }}\left(1-\alpha_{s}\right)\left(1-\alpha_{c}\right) .
$$

(v) Ceiling reflection of right wall and left wall groups B2, B3 combined with group A4 to produce groups D6 and $\mathrm{C} 8$ in Figure $3 ; x=W, y=H$

$$
p_{w}^{2}(W, H)_{\text {incident reverb }}\left(1-\alpha_{s}\right)\left(1-\alpha_{c}\right) .
$$

(vi) Higher order reflections $(m \geq 2)$ are given by a factor $F$ to be multiplied by the total of the eight reflection groups above

$$
F=1+\sum_{m=2}^{\infty} \frac{\left(1-\alpha_{c}\right)^{m}\left(1-\alpha_{f}\right)^{m}\left(1-\alpha_{s}\right)^{2 m}}{m^{2}},
$$

where $M$ higher order groups are used for numerical calculation.

For the worst case of all absorption coefficients equal to zero, $F$ is a convergent series given by

$$
F=\sum_{m=1}^{\infty} \frac{1}{m^{2}}=\frac{\pi^{2}}{6} .
$$

The total mean square pressure incident to the front wall is given by summing the six components above from (8)-(14). It is given for $x=0, y=0$ at the center of the cab wall. Variations at other locations over the wall will be minimal due to the uniform distribution of sound power considered from the machine radiating surface and the multiple reflections in the volume. Consider

$$
\begin{aligned}
& p_{w \text { incident reverb }}^{2} \\
& =p_{w}^{2}(0,0)_{\text {incident reverb }} \\
& +\left[p_{w}^{2}(0, H)_{\text {incident reverb }}+p_{w}^{2}(0, H)_{\text {incident reverb }}\left(1-\alpha_{c}\right)\right. \\
& +2 p_{w}^{2}(W, 0)_{\text {incident reverb }}\left(1-\alpha_{s}\right) \\
& +2 p_{w}^{2}(W, H)_{\text {incident reverb }}\left(1-\alpha_{c}\right) \\
& \left.+2 p_{w}^{2}(0, H)_{\text {incident reverb }}\left(1-\alpha_{c}\right)\left(1-\alpha_{c}\right)\right] F .
\end{aligned}
$$

The total incident pressure squared is the sum of (4) and (16). Consider

$$
p_{w \text { total incident }}^{2}=p_{w \text { inciden direct }}^{2}+p_{w \text { incident reverb }}^{2} .
$$

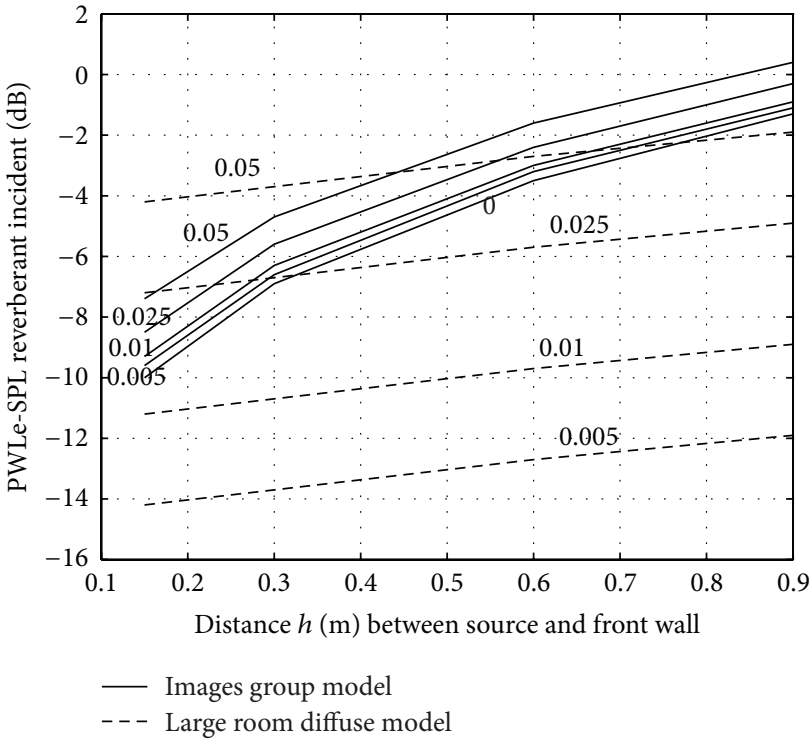

FIGURE 5: Relative reverberant contribution to hard wall case. Values on the curves are alpha hard absorption coefficients $0,0.005,0.01$, 0.025 , and 0.05 .

\section{Numerical Examples}

Numerical examples with $W=2.4 \mathrm{~m} ., H=1.8 \mathrm{~m}$., and $h=0.15 \mathrm{~m} ., 0.30 \mathrm{~m} ., 0.60 \mathrm{~m}$., and $0.90 \mathrm{~m}$. are used to compare the predictions from the images group model where all hard surfaces have an absorption coefficient of zero to a large room diffuse field model where hard surfaces have absorption coefficients of $0.005,0.01,0.025$, and 0.05 . The reduction of the reverberant and total sound pressure levels incident to the front wall from a completely hard wall enclosure to one with the absorption coefficient of the front wall equal to 0.8 are determined.

The large room diffuse field reverberant model is the common result as given in Embleton [6]. Consider

$$
\begin{gathered}
p_{w \text { incident reverb }}^{2}=\frac{\rho c I I_{e}}{S \bar{\alpha}} \\
\left.S \bar{\alpha}=[W H+2 h(W+H)] \alpha_{\text {hard }}+W H \alpha_{\text {front wall }}\right] .
\end{gathered}
$$

The total incident pressure for the large room diffuse field reverberant model is the sum of the direct and reverberant components and from (4), it is given as

$$
p_{w \text { Total incident }}^{2}=\rho c I I_{e}\left(\frac{1}{H W}+\frac{1}{S \bar{\alpha}}\right)
$$

Figure 5 gives a comparison of the difference between incident reverberant sound pressure level $\left(\mathrm{SPL}_{\text {incident reverb }}\right)$ 


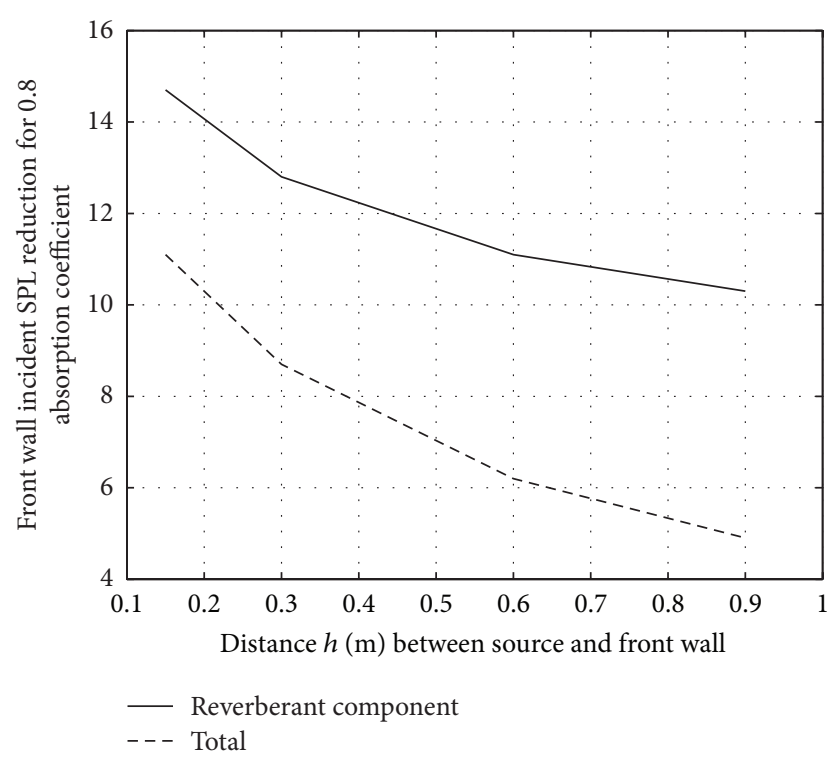

FIGURE 6: Images group model predictions of incident sound level. Total is the sum of direct and reverberant components.

and machine surface sound power level $\left(\mathrm{PWL}_{e}\right)$. It is determined by rewriting the incident reverberant pressure in (5) in terms of a factor $\beta$. Consider

$$
\begin{aligned}
& p_{w}^{2}(x, y)_{\text {incident reverb }} \\
& \quad=\rho c I I_{e} \beta \longrightarrow \mathrm{SPL}_{\text {incident reverb }}-\mathrm{PWL}_{e} \\
& \quad=10 \log _{10}\left(\frac{\rho c I I_{\mathrm{ref}} \beta}{p_{\mathrm{ref}}^{2}}\right) .
\end{aligned}
$$

Figure 5 gives a comparison of the $\mathrm{SPL}_{\text {incident reverb }}-$ $\mathrm{PWL}_{e}$ for the images group and large room diffuse models. Figure 6 gives the noise reduction results for the image model. As the distance $h$ between the source plane and the front wall decreases, both the reverberant contribution and the total reduction increase because the reverberant contribution increases as one would expect. Table 1 gives the noise reduction results for the large room model. For both Figure 6 and Table 1, total refers to the combination of the incident direct and reverberant contributions. Note that $\mathrm{SPL}_{\text {incident reverb }}-$ $\mathrm{PWL}_{e}$ and the noise reductions are essentially independent of the distance $h$ in the 0.15 to $0.90 \mathrm{~m}$. range because $h$ is small enough compared to $H$ and $W$ as to not significantly influence the large room diffuse model product $S \bar{\alpha}$. Also, the large room diffuse model reductions increase with decreasing $\alpha_{\text {hard }}$ which is expected, but this requires an accurate value of the hard wall sound absorption coefficient typically obtained with a measurement. The amount of reductions predicted from the two models can be significantly different if the sound absorption of hard wall surfaces is estimated. Furthermore, very small hard wall surface absorption values can lead to unrealistically high estimates. The low sensitivity of the images group model results to hard wall sound absorption coefficient values indicates that an initial absorption measurement of a hard wall condition is not required with
TABLE 1: Large room diffuse model predictions of incident sound level reductions.

\begin{tabular}{lcc}
\hline $\begin{array}{l}\text { Sound } \\
\text { absorption } \\
\begin{array}{l}\text { coefficient of } \\
\text { hard surfaces }\end{array}\end{array}$ & $\begin{array}{c}\text { Front wall incidence SPL } \\
\text { reduction for } \\
\text { revberant part for each } \\
h \text { value from } 0.15 \text { to } \\
0.90 \mathrm{~m} .\end{array}$ & $\begin{array}{c}\text { Front wall incidence } \\
\text { SPL reduction for } \\
\text { total for each } h \text { value } \\
\text { from } 0.15 \text { to } 0.90 \mathrm{~m} .\end{array}$ \\
\hline 0.005 & 18.5 & 18.0 \\
0.01 & 15.6 & 15.0 \\
0.025 & 11.6 & 10.1 \\
0.05 & 8.8 & 8.3 \\
\hline
\end{tabular}

the images group model. A value of zero for the images group model can be used for an enclosure defined as containing hard walls. Thus the utility of the images group model for estimating noise reduction due to adding an absorptive lining for this class of enclosure with initially all hard internal surfaces can be relevant, especially, as it does not require a specific value for the sound absorption coefficient of the hard surfaces. The low sensitivity of the images group model to hard wall absorption coefficient enhances the reliability of the method for the close-fitting enclosures described here. As shown in Figures 5 and 6 and Table 1, this feature can be expected to provide more accurate estimates than the large room diffuse field model to predict the effect of adding high sound absorption material to provide noise reduction. Also, the use of zero hard wall sound absorption in the images group model will give a more conservative estimate than the large room diffuse field model with very low absorption values will give. This provides increased risk mitigation for meeting noise reduction criteria by adding sound absorption material to the enclosure. Another interesting result seen in Figure 5 is the images group model hovers (in an average sense across source, wall distances) around the large room diffuse field model for 0.05 hard wall absorption coefficient. The 0.05 value has been used quite often by the author and others in estimating hard wall conditions while using the large room diffuse field model. One of many important examples of this class of enclosure is a large diesel engine enclosure or a diesel engine cab on a locomotive where the source is the relatively flat-like surface of the side of the engine and where the cab wall is close to it. The material is usually thick steel or solid fiberglass and coupled with the hard surfaces of engine side, cab bulkheads, cab floor, and ceiling; the situation closely matches the geometry and conditions of this paper.

\section{Conclusions}

The mathematical images group model presented here provides a means of estimating the internal sound level and noise reduction of a tight-fitting enclosure without having to estimate or measure the initial hard wall sound absorption. It provides a means of estimating the internal sound level as it varies with distance from machine surface to enclosure surface, a factor that should be important for tight-fitting enclosures. The results show that the behavior of standard 
large room diffuse field model does not provide this variance and is very sensitive to initial absorption conditions. The key features of the images group model are the use of Hohenwarter [8] machine surface radiation in the context of combining it with the small region's main reverberation component between machine and enclosure as an image sum. The main reverberation component is viewed as a discrete line-type source and then extended in the sense of its images to provide the contribution of other surfaces. Also, the low sensitivity to hard wall sound absorption coefficient allows the use of zero sound absorption values for the initial hard surfaces case and precludes having to apply an educated but quite possibly an inaccurate estimate or actual measurement of the hard surfaces condition. Convergence of the particular images group sum method developed here for zero hard wall absorption allows this preclusion. The results indicate that the images group model presents a method that can provide more accurate and reliable estimates than the large room diffuse model for the internal noise level in the type of close-fitting enclosure and noise source presented.

\section{Conflict of Interests}

The author declares that there is no conflict of interests regarding the publication of this paper.

\section{References}

[1] L. W. Tweed and D. T. Tree, "Three methods for predicting the insertion loss of close-fitting acoustical enclosures," Noise Control Engineering, vol. 10, no. 2, pp. 74-79, 1978.

[2] R. S. Jackson, "Some aspects of the performance of acoustic hoods," Journal of Sound and Vibration, vol. 3, no. 1, pp. 82-94, 1966.

[3] M. C. Junger, "Sound transmission through an elastic enclosure acoustically coupled to a noise source," ASME Paper No. 70WA/DE-12, 1970.

[4] I. L. Ver, "Reduction of noise by acoustic enclosures," in Proceedings of the ASME Design Engineering Technical Conference, Cincinnati, Ohio, USA, 1973.

[5] T. A. Osman, "Design charts for the selection of acoustical enclosures for diesel engine generator sets," Proceedings of the Institution of Mechanical Engineers A: Journal of Power and Energy, vol. 217, no. 3, pp. 329-336, 2003.

[6] T. F. Embleton, "Sound in large rooms," in Noise and Vibration Control, L. L. Beranek, Ed., chapter 9, McGraw-Hill, New York, NY, USA, 1971.

[7] F. Sgard, H. Nelisse, N. Atalla, C. K. Amedin, and R. Oddo, "Prediction of the acoustical performance of enclosures using a hybrid statistical energy analysis: image source model," Journal of the Acoustical Society of America, vol. 127, no. 2, pp. 784-795, 2010.

[8] D. Hohenwarter, "Noise radiation of (rectangular) plane sources," Applied Acoustics, vol. 33, no. 1, pp. 45-62, 1991. 

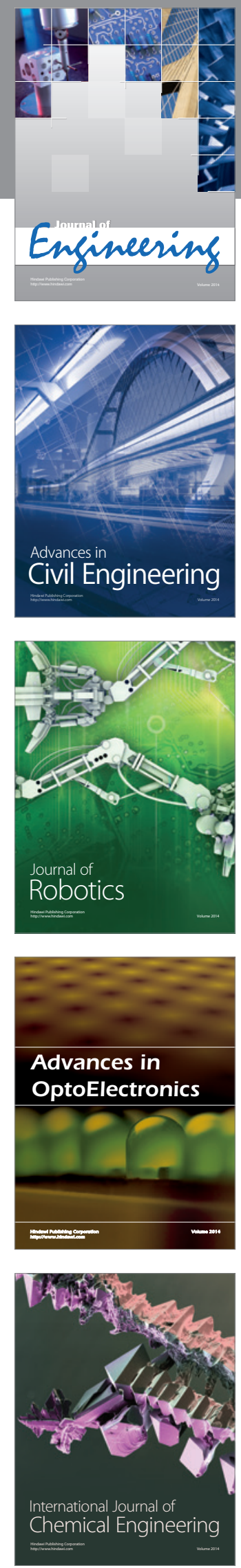

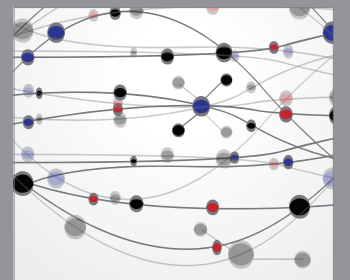

The Scientific World Journal
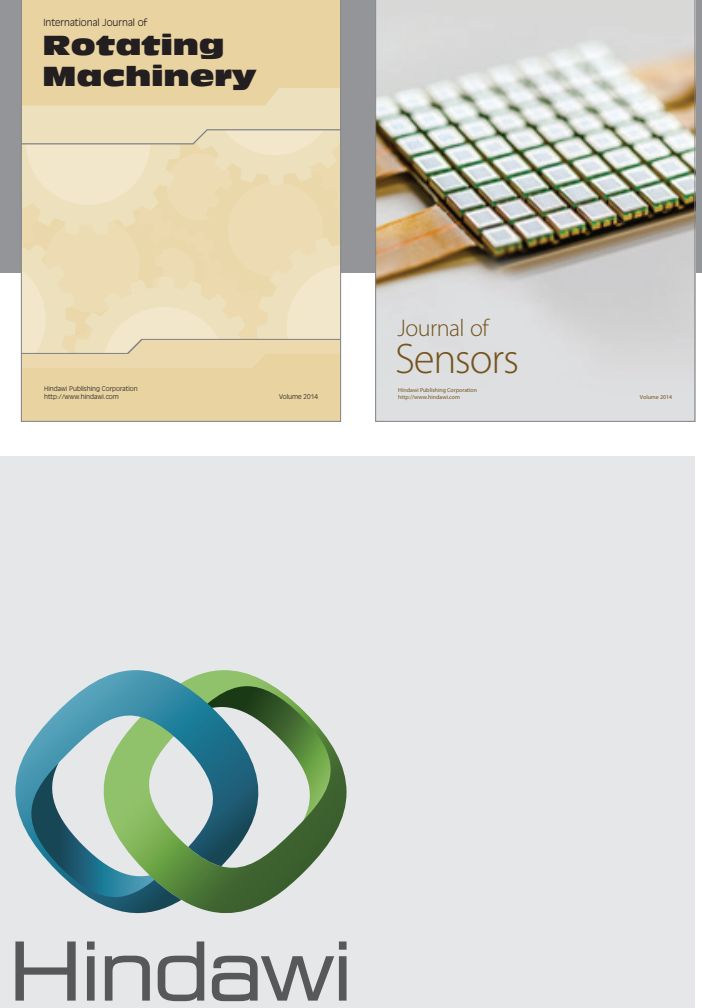

Submit your manuscripts at http://www.hindawi.com
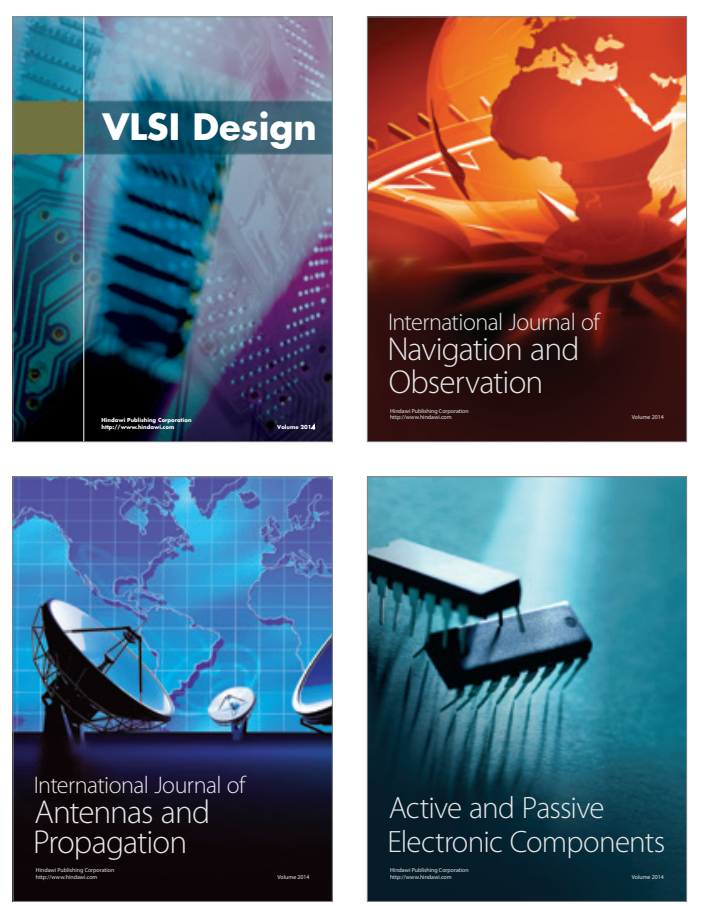
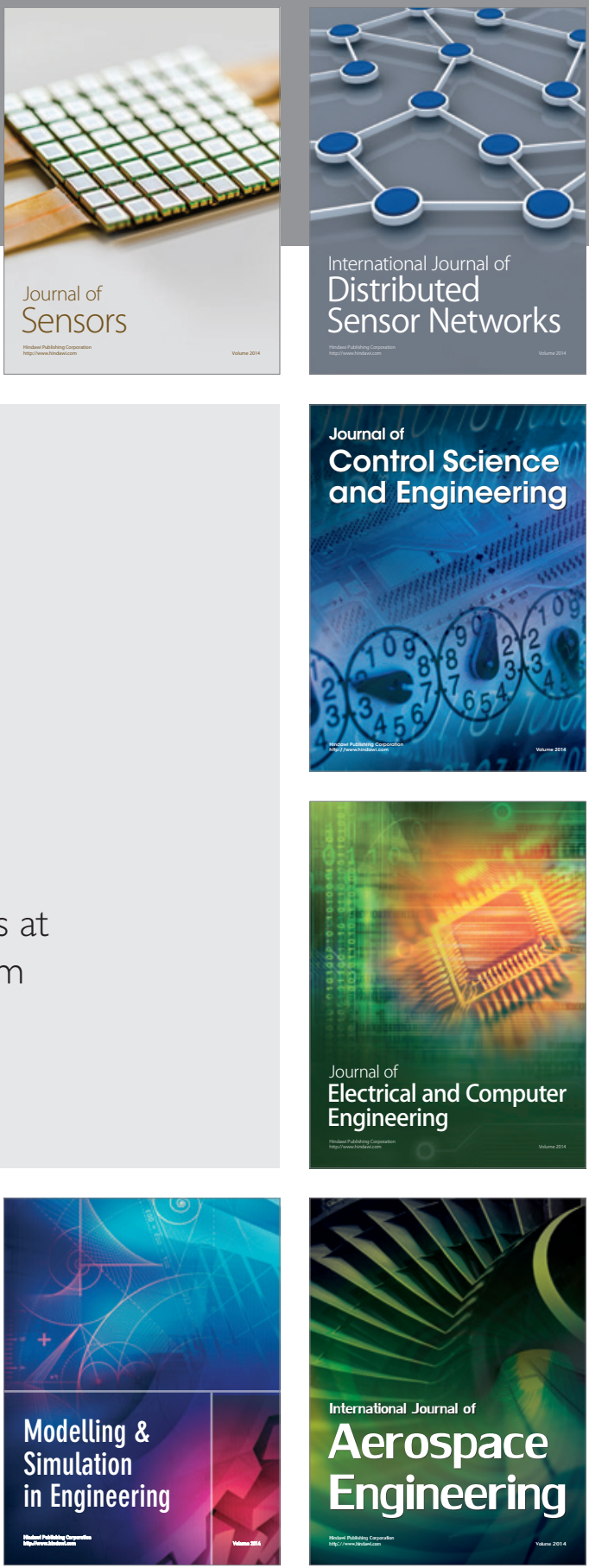

Journal of

Control Science

and Engineering
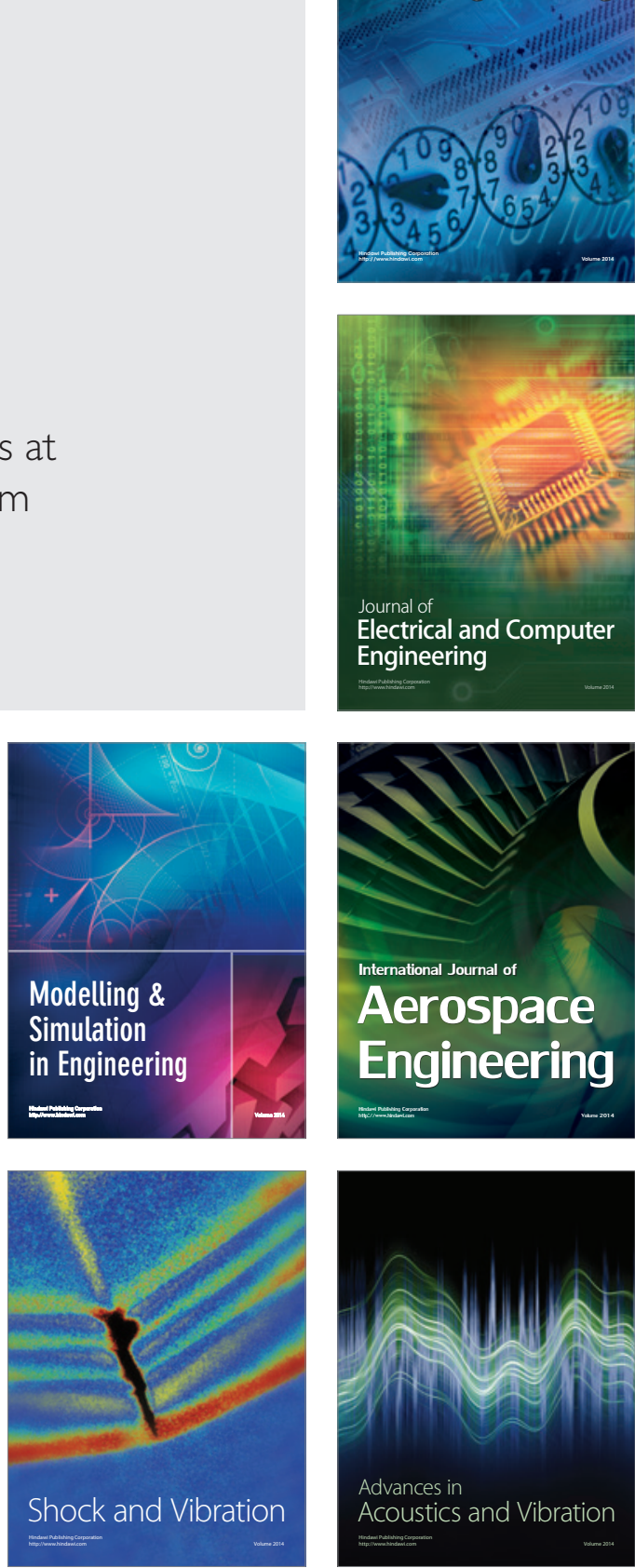